\title{
Simulation of Application Strategies for Local Drug Delivery to the Inner Ear
}

\author{
Stefan K. Plontke ${ }^{a}$ Alec N. Salt ${ }^{b}$ \\ a Tübingen Hearing Research Center and Department of Otorhinolaryngology, Head and Neck Surgery, \\ University of Tübingen, Tübingen, Germany; ${ }^{\mathrm{b}}$ Department of Otolaryngology, Washington University \\ School of Medicine, St. Louis, Mo., USA
}

\section{Key Words}

Drug delivery $\cdot$ Inner ear $\cdot$ Pharmacokinetics $\cdot$ Round window membrane $\cdot$ Computer simulations $\cdot$ Steroids

\begin{abstract}
Local, rather than systemic, drug delivery to the inner ear is becoming more widely used to treat inner ear disorders. While many substances are undergoing preclinical and clinical studies, it is equally important to develop appropriate drug delivery systems. Pharmacokinetic studies are technically demanding in animals and almost impossible in humans. Computer simulations have helped establish the basic principles of drug distribution in the inner ear. The distribution of methylprednisolone in the guinea pig cochlea has been simulated for different drug delivery systems based on kinetic parameters established in prior studies. Results were compared for different rates of drug clearance from the middle ear. Absolute and relative drug levels in the perilymph were highly dependent on how long the drug remained in the middle ear. For a brief (30 min) application, the basal to apical drug gradient was higher than for longer delivery times. These findings show that controlling middle ear drug clearance is of critical importance.
\end{abstract}

Copyright $\odot 2006$ S. Karger AG, Basel

\section{KARGER}

Fax +41613061234

E-Mail karger@karger.ch

www.karger.com
(C) 2006 S. Karger AG, Basel

Accessible online at: www.karger.com/orl

\section{Introduction}

Local drug application to the inner ear is becoming of increasing interest in basic research and in clinics as a more effective delivery route compared to systemic applications [1]. In addition to the identification and testing of substances for the treatment of inner ear disorders, it is important to develop appropriate drug delivery systems and protocols.

There are two main routes for local drug delivery to the inner ear: (1) intracochlear or intravestibular drug delivery, and (2) intratympanic (extracochlear) drug application to the round window membrane (RWM).

At the present time, opening of the human inner ear for the sole purpose of local drug delivery is rarely performed. Intracochlear or intravestibular drug delivery is generally limited to situations where the inner ear is already opened for another reason, such as cochlear implantation [2], or where the applied drug is intended to be ablative [3]. Also drug application into the opened vestibule during stapes surgery might be of interest.

For intratympanic drug delivery to the RWM, a variety of drug delivery systems have been developed for use in humans and animals. Single or repeated intratympanic injections may be performed with (fig. 1b) or without (fig. 1a) visualization of the RWM. Drug application may or may not involve injection onto gelfoam sponges and wicks (fig. 1c) or the use of a hydrogel carrier to stabilize

Dr. Stefan Plontke, Department of Otorhinolaryngology

Head and Neck Surgery, University of Tübingen

Elfriede-Aulhorn-Strasse 5, DE-72076 Tübingen (Germany)

Tel. +4970712988088, Fax +497071293311

E-Mail Stefan.Plontke@uni-tuebingen.de 

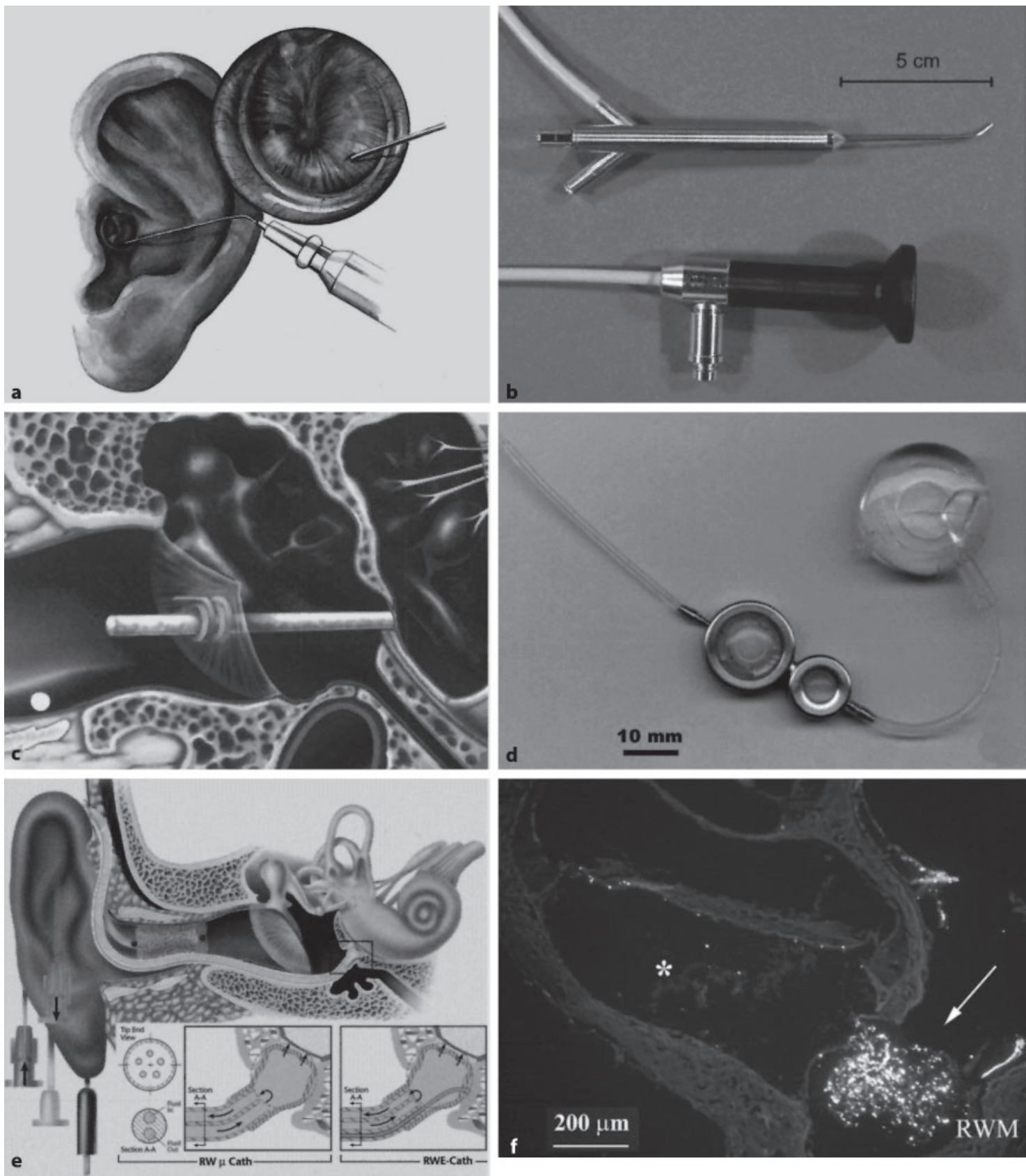

Fig. 1. Application systems for local drug delivery to the inner ear. a Intratympanic injection. b Microotoscope 'Model Tübingen', GYRUS Medical GmbH (former Explorent ${ }^{\circledR} /$ STUEMED $^{\circledR}$ ): outer diameter: $1.2 \mathrm{~mm}$; including an optical channel $(0.5 \mathrm{~mm})$ with 6,000 fibers and two working or suction channels $(0.27$ and $0.3 \mathrm{~mm}$ ), which may be used for targeted intratympanic drug delivery after visualization of the round window niche (from Plontke et al. [27], with permission of S. Karger AG, 2002). c Silverstein Micro Wick ${ }^{\mathrm{TM}}[8]$, printed with permission of Micromedics Inc.,
St. Paul, Minn., USA. d Totally implantable drug delivery system $[14,28]$ (printed with permission of R. Lehner and H.P. Zenner, Germany). e Temporarily implantable catheter approved for intratympanic drug delivery 'Arenberg catheter' (RW $\mu / \mathrm{ECath}^{\mathrm{TM}}$, printed with permission of DURECT TM , Cupertino, Calif., USA). f Poly-lactic/glycolic acid nanoparticles (arrow) loaded with rhodamine entering the scala tympani after placement at the RWM of the guinea pig (from Tamura et al. [11], with permission of Lippincott Williams \& Wilkins, 2005). * = Scala tympani. 
volume [4-8] or the use of biodegradable polymers (fig. 1f) [9-11]. In addition, continuous or discontinuous drug applications may be performed by partly or fully implantable catheters and pump systems (fig. 1d, e) [1216, 29, 30]. For a review, see Salt and Plontke [17].

In addition to aspects such as efficacy, safety and feasibility, the choice of the appropriate delivery system must consider drug pharmacokinetics in the inner ear which is critical to the outcome. Experimental studies of substance distribution in the inner ear are technically challenging due to the small geometry and the difficulty in accessing the inner ear in many species. However, new sampling techniques in combination with quantitative methods for the interpretation of results have led to a basic understanding of substance distribution within the inner ear after local application [17-20,24].

In this article, we present the calculated distributions of methylprednisolone within the scala tympani of the guinea pig after local applications to the RWM using different drug delivery systems. From the results of these simulations, conclusions can be drawn that guide the choice of the appropriate delivery systems for different therapeutic purposes. These findings are relevant to the future development of drug delivery systems for the inner ear.

\section{Methods}

Simulations of glucocorticoid distribution in the cochlear fluids were performed using the Washington University Cochlear Fluids Simulator, version 1.6i. This public-domain computer model is available at http://oto.wustl.edu/cochlea/. The computer program is a finite element model that incorporates many of the passive physical processes by which solutes spread in the inner ear. The program takes into account known parameters such as (1) cross-sectional area of each scala as a function of distance, (2) RWM area and its geometric relationship to the scala tympani, (3) the size of the vestibule, and (4) the rate of substance diffusion as based on molecular weight and temperature. It also incorporates processes with unknown parameters including (5) the rate of substance removal (clearance) from the middle ear, (6) the rate of clearance from each of the inner ear fluid compartments to the systemic blood circulation, (7) the rates of substance exchange between inner ear compartments, and (8) the entry rate of substances through the RWM. 'Clearance' from a specific compartment includes all processes involved in the removal of a substance, including binding, metabolism and movement into the systemic circulation. The use of the simulator and details of the program have been described in detail elsewhere [20,21].

The simulation of glucocorticoid time courses in the scala tympani for different delivery strategies was based on parameters derived from a former interpretation of pharmacokinetic studies in the inner ear after local drug application [22]. Specifically, the following parameters were used for simulating the distribution of methylprednisolone succinate $(50,000 \mu \mathrm{g} / \mathrm{ml}$; molecular weight: $374.48 \mathrm{~g} / \mathrm{ml}$; diffusion coefficient: $0.784 \cdot 10^{-5} \mathrm{~cm}^{2} / \mathrm{s}$ ) after topical application to the RWM in the guinea pig: clearance half-time from scalae to systemic circulation: $130 \mathrm{~min}$; interscala communication half-time: $45 \mathrm{~min}$; RMW permeability: $2 \cdot 10^{-8} \mathrm{~m} / \mathrm{s}$.

The simulated application strategies were as follows. (a) A single intratympanic injection of $100 \mu \mathrm{l}$ of drug solution, a so-called 'one-shot' application. It was assumed that after $30 \mathrm{~min}$ the drug was removed from the middle ear (comparable to the clinical situation with the patient standing up and/or swallowing following injection) so that no further drug would enter the scala tympani through the RWM (fig. 2a). No middle ear clearance was assumed for this condition. (b) A single intratympanic injection of $100 \mu \mathrm{l}$ solution but without volume loss of the applied drug solution over time. This can be described as a 'one-shot' application with volume stabilization. In this case, the entire volume applied was assumed to remain in the middle ear space. Drug movements could occur either from the middle ear into the scala tympani or from the scala tympani into the middle ear depending on the direction of the gradient across the RWM. This scenario was calculated without middle ear clearance (fig. 2c) and for a middle ear clearance of drug with a half-time of $100 \mathrm{~min}$ (fig. 2b). This allowed us to estimate the influence of middle ear clearance on the drug distribution in the cochlear fluids. (c) The third strategy simulated mimicked a continuous delivery of drug to the RWM for 7 days (fig. 2d).

The total simulation time was 2 days (plot axis in fig. $2 \mathrm{a}-\mathrm{c}$ : 1 day) except for the 7-day continuous delivery where substance distribution was simulated for 10 days. All calculations assumed that there was negligible longitudinal volume flow along the scala tympani [23]. For all simulated scenarios, the maximum concentration over the entire application time $\left(\mathrm{C}_{\max }\right)$ and the total dose, calculated by the product of concentration and time (the area under the curve, AUC), were calculated for different locations along the scala tympani (fig. 3).

\section{Results and Conclusions}

The simulations show that longer drug applications to the RWM result in higher absolute drug levels within the inner ear. This is in accordance with recent animal experimental data where longer application of a glucocorticoid to the RWM of the guinea pig resulted in significantly higher drug levels than short application times [18].

For all application scenarios, significant concentration gradients between the basal and apical parts of the cochlea are predicted (fig. 2, 3). The analysis also suggests that brief applications produce the highest drug gradient along the scala tympani with respect to $\mathrm{C}_{\max }$ with minimum drug levels in the apical region. These concentration gradients for methylprednisolone along the scala tympani are qualitatively consistent with those measured recently for a ionic marker (trimethyl phenyl ammonium 


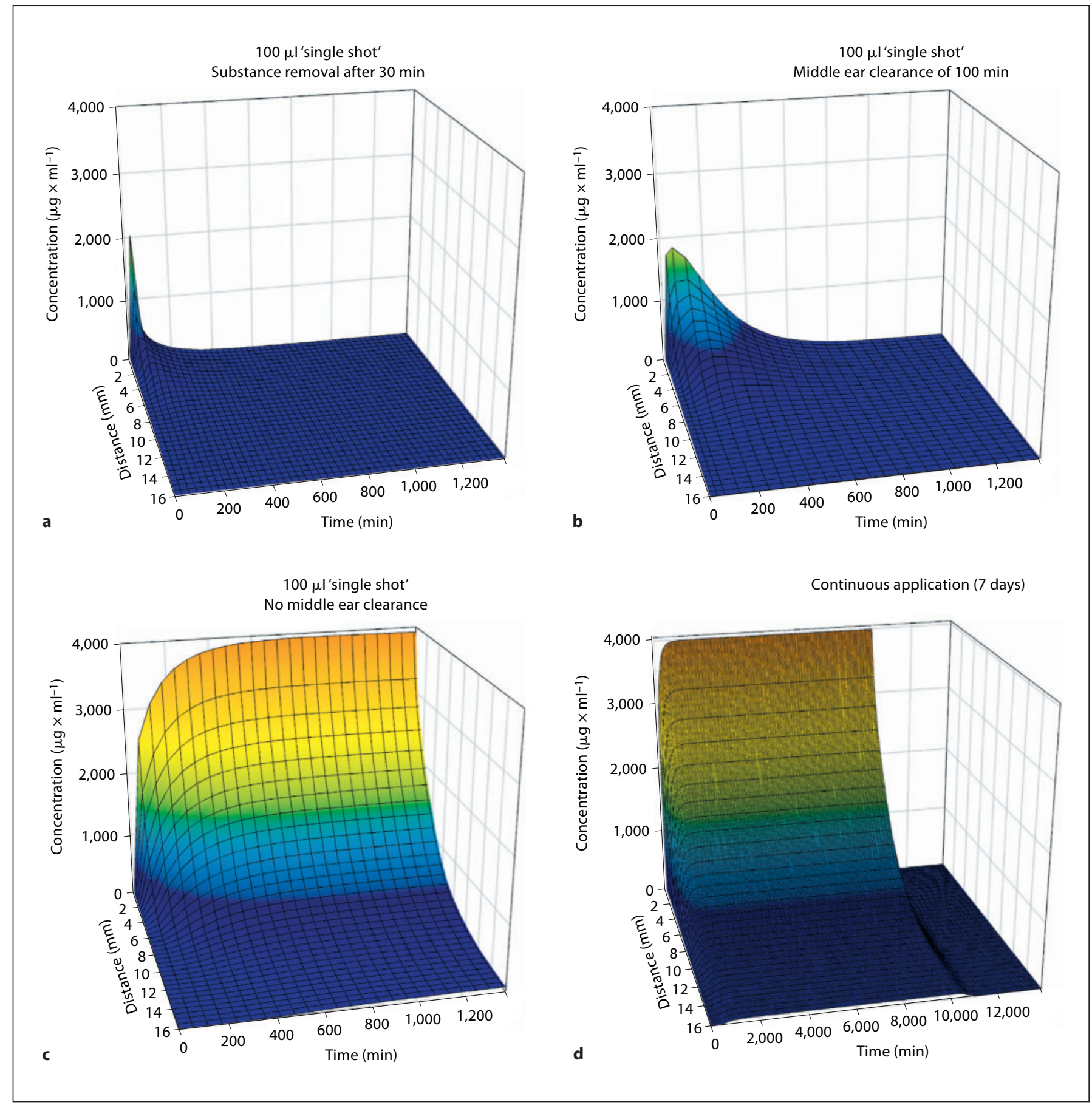

Fig. 2. Summary of calculated methylprednisolone distribution in the scala tympani of the guinea pig as a function of distance and time for four application conditions. Absolute drug levels achieved in the perilymph depend greatly on how long the drug remains in the middle ear. For a brief (30 min) application, the basal/apical gradient is higher than for longer delivery times. The clearly different intracochlear concentration-time curves calculated for different clearance values show the importance of controlling middle ear clearance (b, $\mathbf{c})$. Note that d has a different time axis. 


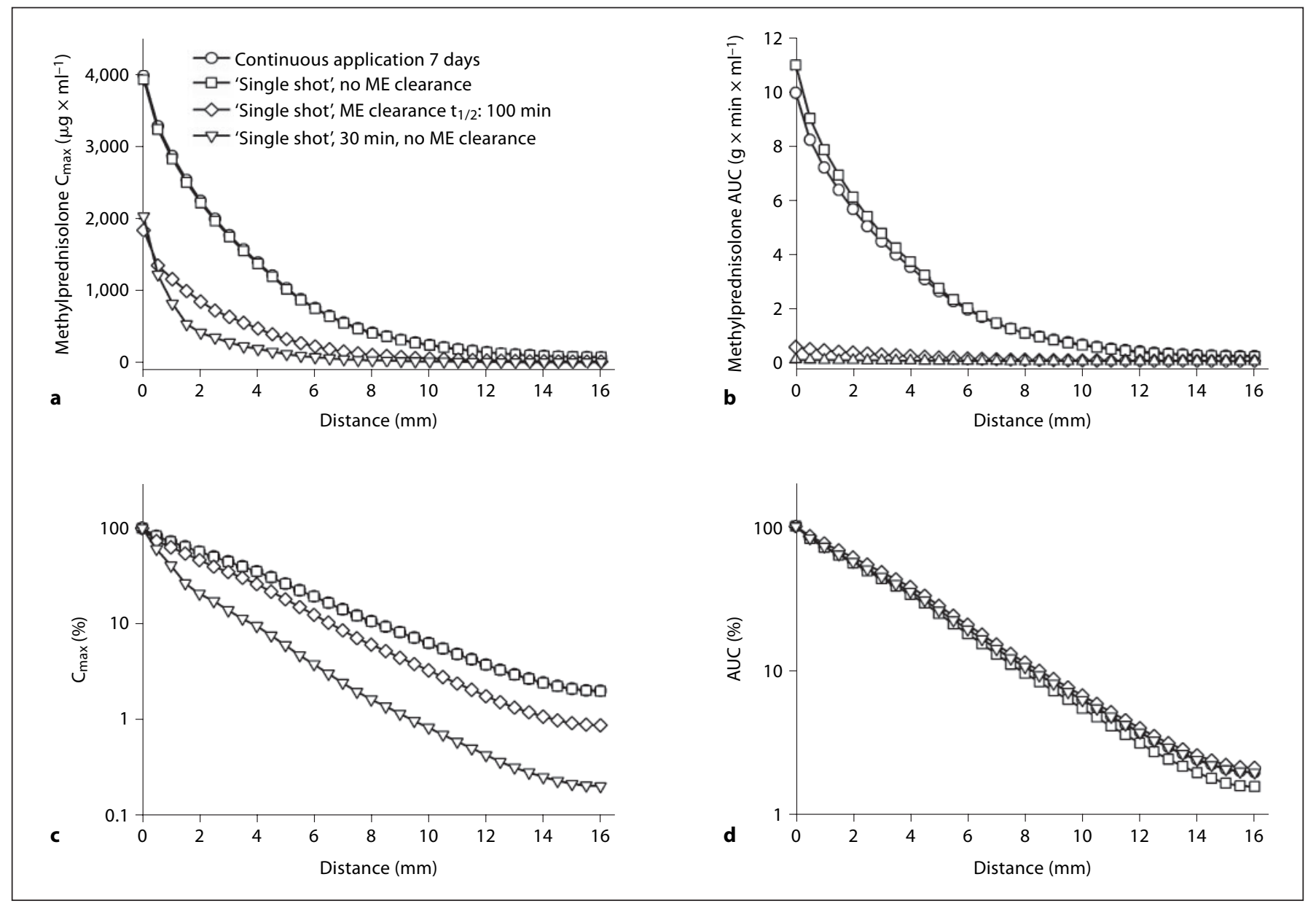

Fig. 3. Comparison of maximum concentration and total dose for different locations in the scala tympani and for different application systems. The physiologic effects of an applied drug depend on whether the ear is most sensitive to the maximum concentration $\left(\mathrm{C}_{\max }\right)$ or whether exposure time and total dose play a role. Calculated $\mathrm{C}_{\max }$ and total dose (AUC) demonstrate a strong dependence of drug level on the time the drug stays in the middle ear. Gradients for $\mathrm{C}_{\max }$ along the scala tympani are greater for brief applications and become markedly reduced with prolonged application. Gradients for AUC are independent of application time. Note logarithmic scale of normalized values in $\mathbf{c}$ and $\mathbf{d}$. ME = Middle ear.

chloride) and for gentamicin by using a novel method of sequential apical sampling $[19,24]$.

In contrast, prolonged drug applications are predicted to result in a more uniform distribution (fig. $3 \mathrm{a}, \mathrm{c})$. On the other hand, comparisons of AUC with different application strategies show that the absolute drug level is altered, but the relative drug distribution with respect to the total dose is little affected (fig. 3b, d). If the drug effects are dominated by $\mathrm{C}_{\max }$, this suggests that prolonged applications may be more appropriate for drugs directed towards auditory function, such as steroid treatments for sudden hearing loss, while drugs directed towards ves- tibular function are more appropriately applied as a oneshot procedure. If the drug effects are dominated by AUC, then the application protocol may have little influence with respect to the relative (but not absolute) drug distribution within the inner ear.

The significantly higher absolute drug levels after longer application times need to be considered when choosing the applied concentration and the drug delivery system.

In addition to investigating the influence of drug application time, the simulations also demonstrate the importance of other physiological processes on inner ear drug levels. Specifically, the presence of middle ear clear- 
ance will affect the drug levels achieved in the perilymph for all single or repeated intratympanic injections of defined drug volumes (fig. $3 \mathrm{a}-\mathrm{c}$ ). If drug is removed from the round window niche (in the example: after $30 \mathrm{~min}$ ), only a small concentration peak is reached very early at the very basal (high frequency) part of the scala tympani. If volume loss of drug is prevented, e.g. through an appropriate carrier system, then the rate of middle clearance is of prime importance, with significantly higher drug levels occurring in the inner ear when the rate of middle ear clearance is slow. This demonstrates the importance of considering the quantification and control of middle ear clearance rates when developing systems for local drug delivery to the inner ear.

The transfer across the RWM barrier limits the amount of drug entering the perilymphatic space. Especially for larger molecules or particles, this has been proven to be of significance as demonstrated by the more efficient gene transfer after intracochlear inoculation as compared to RWM application [25]. Although it may be possible to develop drug additives or procedures to permeabilize the RWM, this limitation may drive the development of safe methods for intracochlear or intravestibular drug application. First results are available using glucocorticoids during cochlear implantation [2].

Computer simulations demonstrate that in local drug delivery to the inner ear, both the drug delivery system and the application protocol significantly influence the distribution of substance within the inner ear.

To select the appropriate drug delivery system, it is therefore necessary to understand and quantify drug distribution in the ear. A major advance in this respect is the development of techniques that allow the quantification of concentration gradients within the scala tympani perilymph after round window drug application $[19,24]$. The goal is to control drug distribution within the inner ear after local application, enabling specific drug targeting to the relevant inner ear structures. Possible ways of reaching this goal are the use of controlled release systems to regulate drug release in the middle ear and possibly methods to manipulate middle ear clearance. Another major step in developing local drug delivery strategies for the inner ear is to overcome or specifically control drug gradients within the cochlea or between the cochlea and the vestibular system. Controlled release carriers applied locally to the inner ear would diffuse in the cochlear fluids without being cleared from them. The carrier would release the drug it was loaded with either through biodegradation or diffusion out of the carrier, a process which could be modulated by external physical stimuli. Other solutions to drug targeting could include immunological methods (using antibodies to direct a drug to a specific cell type) or using magnetic nanospheres [26], the dispersal of which can be influenced by external magnetic fields.

\section{Acknowledgements}

We thank Ruth Gill for assistance in the simulations. This work was supported by grants WK 1-1328-162 and WK 1-1328-171 from BfR/ZEBET (Germany) and grant RO1 DC01368 from NIDCD, NIH (USA). Figure la was created by the artist Andreas Mücke, Berlin, Germany.

\section{References}

1 Lalwani AK, McGuire JF: Pharmacologic treatment of the cochlea and labyrinth; in Cummings CW (ed): Otolaryngology - Head and Neck Surgery. Philadelphia, Mosby Inc, 2005, vol 4, pp 2944-2976.

-2 DeCeulaer G, Johnson S, Yperman M, Daemers K, Offeciers FE, O’Donoghue GM, Govaerts PJ: Long-term evaluation of the effect of intracochlear steroid deposition on electrode impedance in cochlear implant patients. Otol Neurotol 2003;24:769-774.

$\checkmark 3$ Schuknecht HF: Ablation therapy for the relief of Ménière's disease. Laryngoscope 1956; 66:859-870.

-4 Arriaga MA, Goldman S: Hearing results of intratympanic steroid treatment of endolymphatic hydrops. Laryngoscope 1998;108: 1682-1685.
5 Gouveris H, Selivanova O, Mann W: Intratympanic dexamethasone with hyaluronic acid in the treatment of idiopathic sudden sensorineural hearing loss after failure of intravenous steroid and vasoactive therapy. Eur Arch Otorhinolaryngol 2005;262:131134.

6 Kroath F: Transtympanale Injektion zur Behandlung des Ménièreschen Syndroms. Z Laryngol Rhinol Otol 1960;39:190-195.

-7 Silverstein H, Arruda J, Rosenberg SI, Deems D, Hester TO: Direct round window membrane application of gentamicin in the treatment of Ménière's disease. Otolaryngol Head Neck Surg 1999;120:649-655.
8 Silverstein H: Use of a new device, the MicroWick, to deliver medication to the inner ear. Ear Nose Throat J 1999;78:595-598, 600.

\$9 Arnold W, Senn P, Hennig M, Michaelis C, Deingruber K, Scheler R, Steinhoff HJ, Riphagen F, Lamm K: Novel slow- and fasttype drug release round-window microimplants for local drug application to the cochlea: an experimental study in guinea pigs. Audiol Neurootol 2005;10:53-63.

10 Endo T, Nakagawa T, Kita T, Iguchi F, Kim TS, Tamura T, Iwai K, Tabata Y, Ito J: Novel strategy for treatment of inner ears using a biodegradable gel. Laryngoscope 2005;115: 2016-2020 
-11 Tamura T, Kita T, Nakagawa T, Endo T, Kim TS, Ishihara T, Mizushima Y, Higaki M, Ito J: Drug delivery to the cochlea using PLGA nanoparticles. Laryngoscope 2005; 115: 2000-2005.

12 Brown JN, Miller JM, Altschuler RA, Nuttall AL: Osmotic pump implant for chronic infusion of drugs into the inner ear. Hear Res 1993;70:167-172.

13 Chen Z, Kujawa SG, McKenna MJ, Fiering JO, Mescher MJ, Borenstein JT, Leary Swan EE, Sewell WF: Inner ear drug delivery via a reciprocating perfusion system in the guinea pig. J Control Release 2005;110:1-19.

14 Praetorius M, Limberger A, Muller M, Lehner R, Schick B, Zenner HP, Plinkert P, Knipper $\mathrm{M}$ : A novel microperfusion system for the long-term local supply of drugs to the inner ear: implantation and function in the rat model. Audiol Neurootol 2001;6:250-258.

15 Plontke S, Lowenheim H, Preyer S, Leins P, Dietz K, Koitschev A, Zimmermann R, Zenner HP: Outcomes research analysis of continuous intratympanic glucocorticoid delivery in patients with acute severe to profound hearing loss: basis for planning randomized controlled trials. Acta Otolaryngol 2005; 125:830-839.

16 Thomsen J, Charabi S, Tos M: Preliminary results of a new delivery system for gentamicin to the inner ear in patients with Ménière's disease. Eur Arch Otorhinolaryngol 2000; 257:362-365
17 Salt AN, Plontke SK: Local inner ear drug delivery and pharmacokinetics. Drug Discov Today 2005;10:1299-1306.

18 Hahn H, Kammerer B, DiMauro A, Salt AN, Plontke SK: Cochlear microdialysis for quantification of dexamethasone and fluorescein entry into scala tympani during round window administration. Hear Res 2006;212:236-244.

19 Mynatt R, Hale S, Gill R, Plontke S, Salt AN: Demonstration of a longitudinal concentration gradient along scala tympani by sequential sampling of perilymph from the cochlear apex. J Assoc Res Otolaryngol 2006;7:182193.

20 Plontke SK, Wood AW, Salt AN: Analysis of gentamicin kinetics in fluids of the inner ear with round window administration. Otol Neurotol 2002;23:967-974.

21 Salt AN: Simulation of methods for drug delivery to the cochlear fluids. Adv Otorhinolaryngol 2002;59:140-148.

22 Plontke SK, Salt AN: Quantitative interpretation of corticosteroid pharmacokinetics in inner ear fluids using computer simulations. Hear Res 2003;182:34-42.

23 Salt AN, Ma Y: Quantification of solute entry into cochlear perilymph through the round window membrane. Hear Res 2001;154:8897.
24 Plontke SK, Mynatt R, Hale S, Salt AN: Gentamicin concentration gradient along scala tympani of the guinea pig cochlea after round window drug administration. Assoc Res Otolaryngol Abs 2006:29.

25 Stover T, Yagi M, Raphael Y: Cochlear gene transfer: round window versus cochleostomy inoculation. Hear Res 1999;136:124130.

26 Kopke RD, Wassel RA, Mondalek F, Grady B, Chen K, Liu J, Gibson D, Dormer KJ: Magnetic nanoparticles: inner ear targeted molecule delivery and middle ear implant. Audiol Neurootol 2006;11:123-133.

27 Plontke SK, Plinkert PK, Plinkert B, Koitschev A, Zenner HP, Lowenheim $\mathrm{H}$ : Transtympanic endoscopy for drug delivery to the inner ear using a new microendoscope. Adv Otorhinolaryngol 2002;59:149155.

28 Lehner R, Brugger H, Maassen MM, Zenner HP: A totally implantable drug delivery system for local therapy of the middle and inner ear. Ear Nose Throat J 1997;76:567-570.

29 Kopke RD, Hoffer ME, Wester D, O'Leary MJ, Jackson RL: Targeted topical steroid therapy in sudden sensorineural hearing loss. Otol Neurotol 2001;22:475-479.

30 Plontke SK, Zimmermann R, Zenner HP Löwenheim $\mathrm{H}$ : Technical note on microcatheter implantation for local inner ear drug delivery: Surgical technique and safety aspects. Otol Neurotol 2006;27:912-917. 